Journal of Bangladesh Academy of Sciences, Vol. 37, No. 1, 83-91, 2013

\title{
EFFECT OF THICKNESS ON THE OPTICAL PROPERTIES OF GaAs THIN FILMS
}

\author{
CHITRA DAS ${ }^{*}$, JAHANARA BEGUM ${ }^{1}$, TAHMINA BEGUM ${ }^{1}$ AND SHAMIMA \\ CHOUDHURY
}

Department of Physics, University of Dhaka, Dhaka-1000, Bangladesh

\begin{abstract}
Effect of thickness on the optical and electrical properties of gallium arsenide (GaAs) thin films were studied. The films of different thicknesses were prepared by vacuum evaporation method $\left(\sim 10^{-4} \mathrm{~Pa}\right)$ on glass substrates at a substrate temperature of $323 \mathrm{~K}$. The film thickness was measured in situ by a frequency shift of quartz crystal. The thicknesses were 250, 300 and $500 \mathrm{~nm}$. Absorption spectrum of this thin film had been recorded using UV-VIS-NIR spectrophotometer in the photon wavelength range of $300-2500 \mathrm{~nm}$. The values of some important optical parameters of the studied films (absorption coefficient, optical band gap energy and refractive index; extinction co-efficient and real and imaginary parts of dielectric constant) were determined using these spectra. Transmittance peak was observed in the visible region of the solar spectrum. Here transmittance showed better result when thicknesses were being increased. The optical band gap energy was decreased by the increase of thickness. The refractive index increased by increasing thickness while extinction co-efficient and real and imaginary part of dielectric constant decreased.
\end{abstract}

Key words: Thickness, Optical properties, GaAs thin films

\section{INTRODUCTION}

Semiconductor materials are very important in the study of scientific and technology spaces such as rectifiers, transistors, laser diodes and optical filters. The semiconductor family consists of many elementary and binary compounds. As they are technologically important, there are vast studies most of them and their combinations in the form of alloys (Francoeur et al. 2003).

Gallium arsenide (GaAs) is an III-V compound semiconductor which is suitable for electronic and optoelectronic devices. The thin films of GaAs are used in the manufacture of light emitting diodes (LEDs), field effect transistors, integrated circuits (ICs), solar cells. It is also useful in ultra high radio frequency, switching applications, detection of $\mathrm{X}$-rays. GaAs is considered as a prospective competitor of silicon because of its wider band gap and higher electron mobility. This is a direct band gap material. The band gap of $\mathrm{GaAs}$ is $1.42 \mathrm{eV}$ which is very near to the optimum band gap energy $1.5 \mathrm{eV}$ for solar energy conversion (Brozel and Stillman 1996). Its electron effective mass is only 1/16 of

*Corresponding author: <dchitra20@yahoo.com>.

${ }^{1}$ Experimental Physics Division, Atomic Energy Centre, Dhaka-1000, Bangladesh. 
that of silicon $(\mathrm{Si})$. This is why GaAs becomes a likely candidate for the production of future integrated circuits despite its technologically more elaborate processing (Kayali and Ponchak 1996).

The effect of rate of deposition on the optical parameters of GaAs thin films was studied which were grown by thermal evaporation method (Majeed 2011). The transmission, optical energy gap, extinction co-efficient, real and imaginary dielectric constant increased while refractive index decreased with the increase of rate of deposition. Hydrogen-doped GaAs thin films were made by rf magnetron sputtering technique and their structural, optical and electrical properties were studied (Yan-Ping et al. 2008). The bonded hydrogen content was increased with increasing partial pressure $\mathrm{P}_{\mathrm{H}}$ of $\mathrm{H}_{2}$. There hydrogen addition was shifted the optical absorption edge to higher energies, decreased the dark conductivity and improved the photo-sensitivity. The polycrystalline GaAs on quartz substrate was deposited at higher temperature, $400^{\circ} \mathrm{C}$ and the growth technique was molecular beam epitaxy (Mosaddequr et al. 2002). Films deposited at a relatively higher temperature $\left(400^{\circ} \mathrm{C}\right)$ showed high crystalline quality with high surface roughness. Growth at a lower temperature $\left(250^{\circ} \mathrm{C}\right)$ and subsequent annealing at high temperature yielded GaAs film of smooth surface and high crystalline quality with crystals oriented predominantly towards the (111) plane. The optical absorption and photoconductivity of $50 \mathrm{~nm}$ ultra thin films of single crystal GaAs by selective etching of epitaxial layers grown by molecular beam epitaxy were measured (Halliday et al. 1995). The optical absorption showed a large absorption below the fundamental band edge due to Franz-Keldysh shift. Electrical conductivity and temperature dependence on GaAs thin films deposited by RF sputtering were measured (Tsuji et al. 1992). The conductivity was in the range $10^{-4} \Omega^{-1} \mathrm{~cm}^{-1}$. Optical and transport properties of $\mathrm{p}$ - and n-type GaAs were investigated (Chowdhury et al. 2012, Sharmin et al. 2012).

The low intrinsic carrier density of GaAs in a pure form indicates that GaAs is intrinsically a very poor conductor and is commonly referred to as being semi-insulating. This semi-insulting property allows many active devices to be grown on a single substrate, where the semi-insulating GaAs provides the electrical isolation of each device; an important feature in the miniaturization of electronic circuitry. GaAs is very resistant to radiation damage. This, along with its high efficiency makes GaAs very desirable for outer space applications.

Present work reports the effect of film thickness on optical properties of vacuum evaporated thin films of GaAs via transmittance and reflectance measurements in the wavelength range from 300 to $2500 \mathrm{~nm}$.

Optical parameters such as absorption co-efficient, refractive index, extinction coefficient, dielectric constants were determined. Optical process or absorption method was 
implied to determine the band gap energy. Electrical resistivity and conductivity were also determined.

\section{EXPERIMENTAL DETAILS}

Growth of the films: The samples were prepared by thermal evaporation method using a vacuum evaporation unit type Edwards 306A, UK, in high vacuum $\left(10^{-4} \mathrm{~Pa}\right)$. The thicknesses of the films were 250, 300 and $500 \mathrm{~nm}$ and substrate temperature was $323 \mathrm{~K}$. Glass slides were used as substrates; these were cleaned chemically and ultrasonically. Ntype GaAs powder was used as a source material. The source material is thermally evaporated from a molybdenum boat and the substrate was placed at a distance of $10 \mathrm{~cm}$ from the source. The rate of evaporation for GaAs thin film was $0.2 \mathrm{~nm} / \mathrm{sec}$ measured in situ by the FTM5 quartz crystal thickness monitor (Edwards, UK).

Optical measurements: The optical transmittance, absorption coefficient, the optical band gap energy and the refractive indices of the deposited films were studied by using an UV-VIS-NIR spectrophotometer operated at room temperature.

Transmittance, reflectance and thickness: The variations of optical transmittance $(T \%)$ and absolute specular reflectance $(R \%)$ of the films with wavelength of light incident on them were measured using a dual-beam UV-VIS-NIR recording spectrophotometer (Shimadzu, UV-3100, Japan) in the photon wavelength range from $300-2500 \mathrm{~nm}$. Light signals coming from the samples were detected by an integrating sphere. The thickness of the composite films was checked using an infrared interference method with the spectrophotometer, which depends on the reflectance characteristics of the films. In this method the thickness of a film is given by

$$
d=\frac{\Delta m}{2 \sqrt{n_{1}^{2}-\sin ^{2} \theta}} \frac{1}{\left(1 / \lambda_{1}\right)-\left(1 / \lambda_{2}\right)}
$$

where, $n_{1}$ is the refractive index of the film, $\theta$ is the incident angle of light to the sample, $\lambda_{1}$ and $\lambda_{2}$ are the peak or valley wavelengths in the reflectance spectrum and $\Delta m$ is the number of peaks or valleys between $\lambda_{1}$ and $\lambda_{2}$, where, $\lambda_{2}>\lambda_{1}$.

Absorption coefficient and band gap energy: For transmittance $(T \%)$ at normal incidence and reflectance $(R \%)$ at near-normal incidence of light on the films, expressions for the multiple reflected systems have been /given by (Heavens 1965). Tomlin (1968) simplified these expressions for absorbing films on non-absorbing substrates and expressed them as

$$
\frac{1+R}{T}=\frac{1}{4 n_{2}\left(n_{1}^{2}+k_{1}^{2}\right)}\left[\begin{array}{l}
\left(1+n_{1}^{2}+k_{1}^{2}\right)\left\{\left(n_{1}^{2}+n_{2}^{2}+k_{1}^{2}\right) \cosh 2 \alpha_{1}+2 n_{1} n_{2} \sinh 2 \alpha_{1}\right\} \\
+\left(1-n_{1}^{2}-k_{1}^{2}\right)\left\{\left(n_{1}^{2}-n_{2}^{2}+k_{1}^{2}\right) \cos 2 y_{1}-2 n_{2} k_{1} \sin 2 y_{1}\right\}
\end{array}\right]
$$




$$
\frac{1-R}{T}=\frac{1}{2 n_{2}\left(n_{1}^{2}+k_{1}^{2}\right)}\left[\begin{array}{l}
\left.n_{1}\left(n_{1}^{2}+n_{2}^{2}+k_{1}^{2}\right) \sinh 2 \alpha_{1}+2 n_{1} n_{2} \cosh 2 \alpha_{1}\right\} \\
+k_{1}^{2}\left\{\left(n_{1}^{2}-n_{2}^{2}+k_{1}^{2}\right) \sin 2 y_{1}+2 n_{2} k_{1} \cos 2 y_{1}\right\}
\end{array}\right]
$$

where, $n_{1}$ and $n_{2}$ are the refractive indices of the film and substrate, respectively, $k_{1}$ is the extinction-coefficient of the film, $n_{2}=1.45, \alpha_{1}=\frac{2 \pi k_{1} d}{\lambda}$ and $\quad \gamma_{1}=\frac{2 \pi n_{1} d}{\lambda}$, where, $\lambda$ is the wavelength of light and $d$ is the thickness of the film. Equations (2) and (3) have been solved for $k_{1}$ and $n_{1}$ utilizing a computerized iteration process. The absorption coefficient $\alpha$ was then calculated using $\alpha=\frac{4 \pi k_{1}}{\lambda}$. Dependence of $\alpha$ on photon energy has been analyzed with the existing models discussed subsequently in equation (6) to find the nature and extent of the band gap energy.

Dielectric constant: The dielectric constant was calculated from the following formula:

$$
\begin{aligned}
\varepsilon_{\mathrm{r}} & =\mathrm{n}^{2}-\mathrm{k}^{2} \\
\varepsilon_{\mathrm{i}} & =2 \mathrm{nk}
\end{aligned}
$$

where, $\varepsilon_{\mathrm{r}}$ and $\varepsilon_{\mathrm{i}}$ represented real and imaginary dielectric constant, respectively.

\section{RESULTS AND DISCUSSIONS}

Fig. 1 shows the optical transmittance of 250, 300 and $500 \mathrm{~nm}$ thin films. From these plots we can observe that the increasing of thickness increases transmittance and shifts the peak of transmittance spectrum toward the higher wavelengths (lower energies).

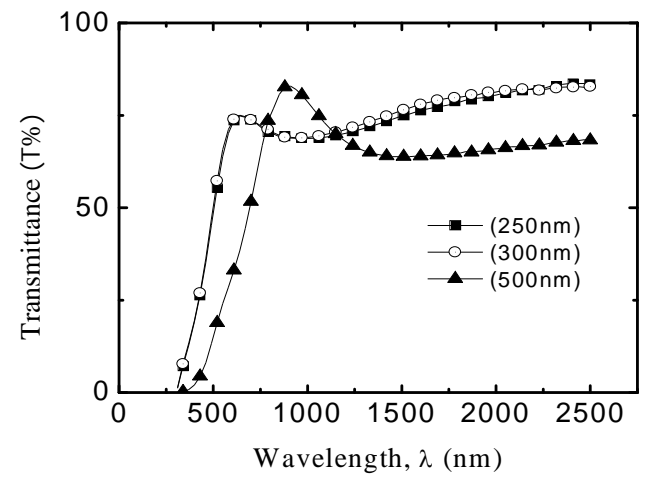

Fig. 1. Dependence of optical transmittance on photon wavelength for GaAs thin films having different thickness.

The transmittance spectrum attains its perfect shape for the sample $500 \mathrm{~nm}$. The shifting of peaks is due to the structural improvement represented by increasing the crystalline grain size (Balkanski and Wallis 2000). The maximum value of T\% was found to be 80 for the film of thickness $500 \mathrm{~nm}$. Indium-doped tin Oxide films showed $\mathrm{T} \%$ as 89 (Chowdhury et al. 2011). 
The behavior of the absorbance spectra is opposite completely to that of the transmittance spectra as shown in Fig. 2. As the thickness increases absorption decreases because of inverse relation between transmission and absorption (Majeed 2011).

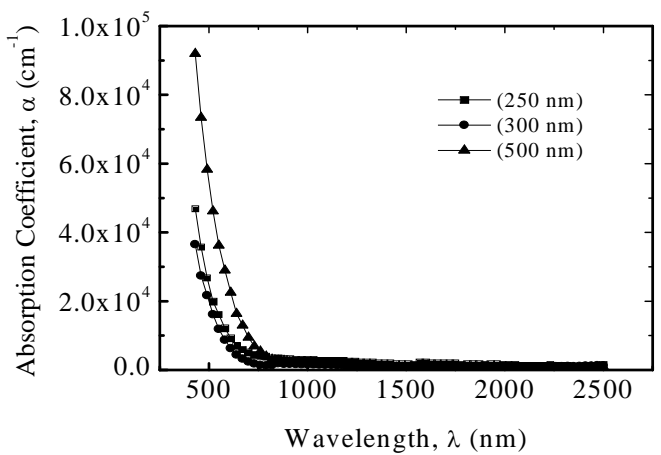

Fig. 2. Dependence of absorption coefficient on photon wavelength for GaAs thin films having different thickness.

The optical band gap $\mathrm{E}_{\mathrm{g}}$ can be estimated from the following relation which is known as the Tauc plot (Tauc 1974):

$$
\alpha h v=A\left(h v-E_{g}\right)^{n}
$$

where, $\mathrm{A}$ is a constant, $v$ is the transition frequency and the exponent $\mathrm{n}$ characterizes the nature of band transition. $\mathrm{n}=1 / 2$ and $3 / 2$ corresponds to direct allowed and direct forbidden transitions and $\mathrm{n}=2$ and 3 corresponds to indirect allowed and indirect

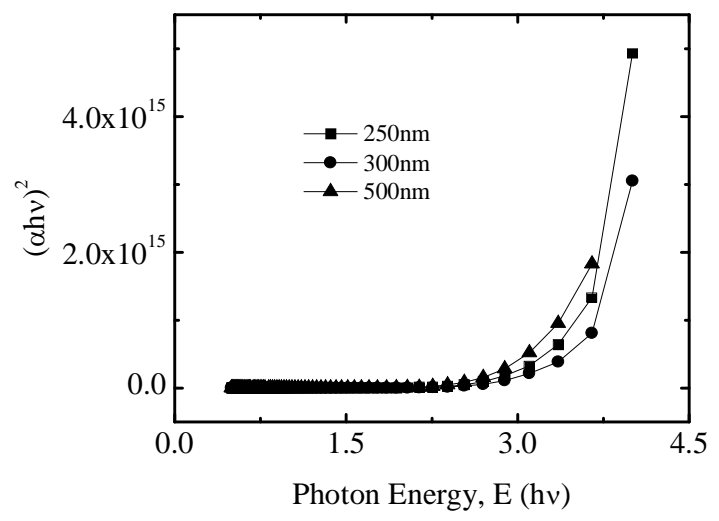

Fig. 3. The plot of $(\alpha h v)^{2}$ vs photon energy for GaAs thin films having different thickness.

forbidden transitions, respectively. The band gap can be obtained from extrapolation of the straight-line portion of the $(\alpha h v)^{1 / n}$ vs hv plot to $h v=0$. It is observed that for all the films, the best straight line is obtained for $n$ equal to $1 / 2$, which is expected for direct allowed transition. 
Fig. 3 shows the plot of $(\alpha h v)^{2}$ vs photon energy. The band gap energies of the samples 250, 300 and $500 \mathrm{~nm}$ films are found to be 3.2, 3.0 and $2.85 \mathrm{eV}$, respectively which is in good agreement with the results of the workers (Halliday et al. 1995). The band gap decreases by the increase of thickness. The Franz-Keldysh shift is the reason for this shift between these data and original band gap (Seegar 1985, Pankove 1971, Keldysh 1958).

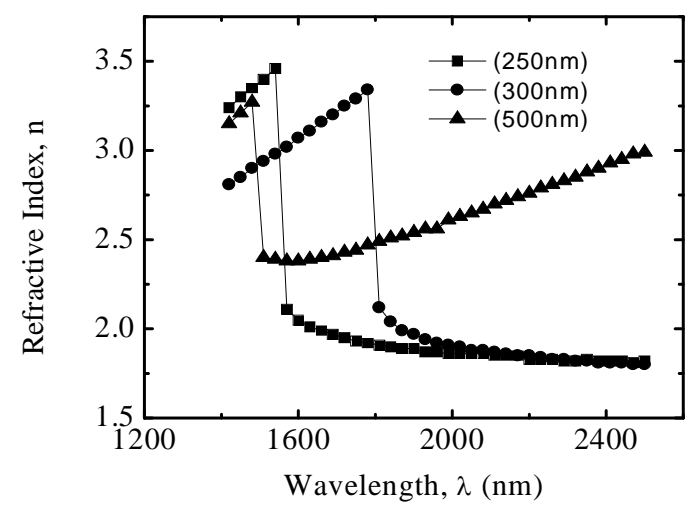

Fig. 4. Dependence of refractive index on photon wavelength for GaAs thin films having different thickness.

From Fig. 4 it is seen that refractive index first increases gradually, attaining a maximum peak they decrease abruptly, finally at higher wavelength range increases for $500 \mathrm{~nm}$ film and decreases exponentially for 250 and $300 \mathrm{~nm}$ film, respectively.

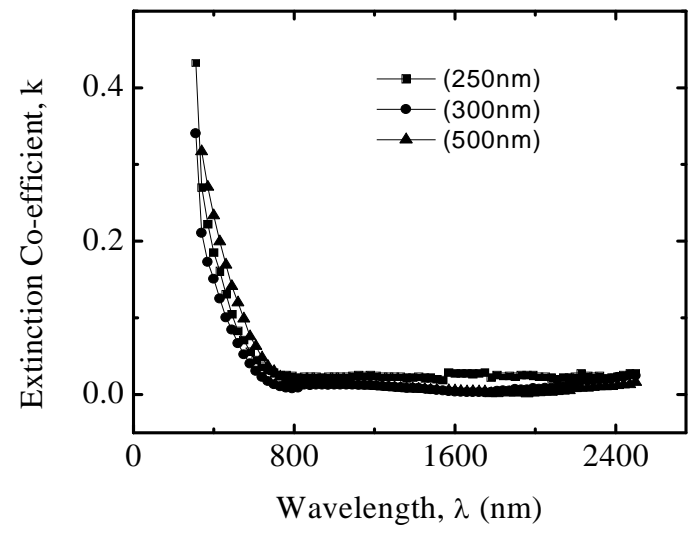

Fig. 5. Variation of extinction co-efficient vs photon wavelength for GaAs thin films having different thickness.

The sharp decrease depends on the surface and volume imperfections. Low refractive index occurs due to successive internal reflections or due to the trapped photon energy within the grain boundary (Ong et al. 2000). 
The dependence of extinction coefficient, $k$, on wavelength, $\lambda$, is shown in Fig. 5. The change in the absorption co-efficient is due to the variation of absorption. The fall in $\mathrm{k}$ may be due to the absorption of light at the grain boundaries. It is almost consistent in higher wavelength region. The low value of $\mathrm{k}$ is a qualitative indication of excellent surface smoothness of the thin films (Bhaskar et al. 2001).

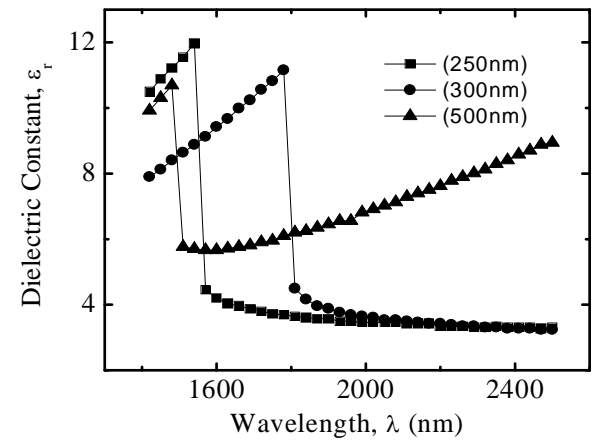

Fig. 6. Real dielectric constant vs photon wavelength for GaAs thin films having different thickness.

From equations 4 and 5 it is seen that real part of dielectric constant denotes refractive index whether imaginary part of dielectric constant denotes $\mathrm{k}$ (Tan and Koughia 2006). So, real and imaginary parts of dielectric constant of GaAs thin films

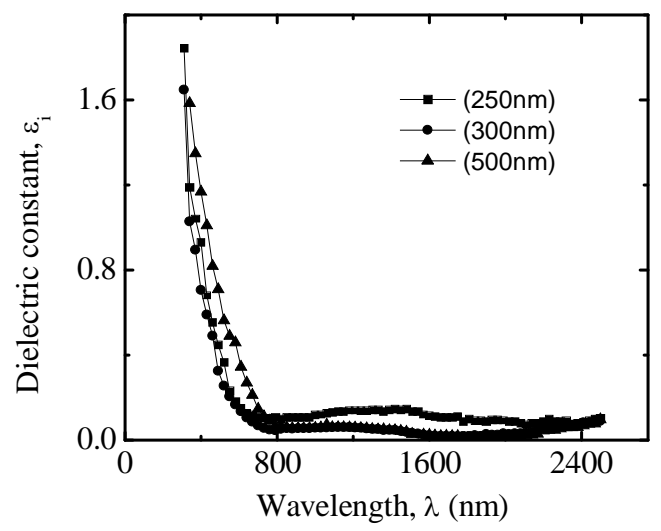

Fig. 7. Imaginary dielectric constant vs photon wavelength for GaAs thin films having different thickness.

show exactly the same behavior of refractive index and k, respectively as shown in Figs 6 and 7. At the anomalous dispersion region the dielectric constant; the real part has a jump or discontinuity. The imaginary part has a peak corresponding to absorption. If the bandwidth of the anomalous dispersion region is very small the jump is seen to be abrupt (Hecht 2002). 


\section{CONCLUSIONS}

From the present work it can be summarized that, with the transmission and absorption spectra the transmission increases by increasing thickness, while absorption decreases. The optical band gap energy decreases with increasing film thickness. The

refractive index increases and $\mathrm{k}$ decreases by increasing film thickness. The real and imaginary part of the dielectric constant behaves similarly as refractive index and $\mathrm{k}$ do, respectively. Finally the room temperature resistivity increased and conductivity decreased with thickness.

\section{ACKNOWLEDGEMENT}

The authors are thankful to Experimental Physics Division, Atomic Energy Center, Dhaka for providing necessary facilities.

\section{REFERENCES}

Balkanski, M. and R.F. Wallis. 2000. Semiconductor Physics and Applications. Oxford University Press.

Bhaskar, S., S. B. Majumder, M. Jain, P. S. Dobal and R. S. Katiyar. 2001. Studies on the Structural, Micro structural and Optical Properties of Sol-gel derived Lead Lanthanum Titanate Thin Films. Mat. Sci. and Eng. B. 87(2): 178-190.

Brozel, M. R. and G. E. Stillman. 1996. Properties of Gallium Arsenide. 3rd Edition. INSPEC. The Institution of Electrical Engineers. London, United Kingdom.

Chowdhury, A.S., S. Choudhury, T. Begum and N. Akhter. 2010. Optical and Transport Properties of GaAs. Dhaka Univ. J. Sci. 58(1): 109-112.

Chowdhury, F.R., S.Choudhury, F. Hasan and T. Begum. 2011. Optical properties of undoped and indium-doped tin Oxide thin films, J. Bangladesh Acad. Sc., 35(1), 99-111.

Francoeur, S., M-J Seong, A. Mascarenhas, S. Tixier, M. Adamcyk and T. Tiedje. 2003. Band Gap of $\mathrm{GaAs}_{1-\mathrm{x}} \mathrm{Bi}_{\mathrm{x}}, 0<\mathrm{x}<3.6 \%$. Appl. Phys. Lett. 82(22): 3874.

Halliday, D. P., J. M. Eggleston, K.Y. Lee, J. E. F. Frost and S. P. Beaumant. 1995. Optical Properties of Ultrathin $50 \mathrm{~nm}$ GaAs membranes. Solid State Commun. 96(6): 359-365.

Heavans, O S. 1965. Optical Properties of Thin Solid Films. Butterworths Scientific Publications, London.

Hecht, E. 2002. Optics. 4th Ed. Addision-Wesely. Chapter 7. pp. 304.

Kayali, S., J. Ponchak and R. Shaw. 1996. GaAs MMIC Reliability Assurance Guide Line for Space Applications. NASA. JPL Publication.

Keldysh, L. V. 1958. The effect of a strong electric field on the optical properties of insulating crystals. Zh. Eksp. Teav. Fiz. 34: 1138-1141 (Translated in Soviet Physics JETP. 34: 788-790), W. Franz and Z. Naturforsch. A13: 484.

Majeed, A. H. 2011. Effect of Rate of Deposition on the Optical Parameters of GaAs Films. Eur. J. Sci. Res. 57(3): 478-484.

Mosaddequr, M. R., S. Tetsuo and J. Takashi. 2002. Ploycrystalline GaAs thin film on Quartz Substrate by Molecular Beam Epitaxy. Presented at $8^{\text {th }}$ International conference on Electronic Materials (IUMRS-ICEM, Xi'an, China). 
Ong, H. C., J. Y. Dai, K. C. Hung, K. C. Hung, Y. C. Chan and S. T. Ho. 2000. Electronic Structures of Polycrystalline $\mathrm{ZnO}$ Thin Films Probed by Electron Energy Loss Spectroscopy. Appl. Phys. Lett. 77(10): 1484.

Pankove, J. I. 1971. Optical Processes in Semiconductors. Courier-Dover Publications.

Seeger, K. 1985. Semiconductor Physics: An Introduction. 9th Ed. Springer.

Sharmin, M., S. Choudhury, N. Akhtar and T. Begum. 2012. Optical and Transport properties of ptype GaAs. J. Bangladesh. Acad. Sci. 36(1): 97-108.

Tan, W. C. and K. Koughia. 2006. Fundamental Optical Properties of Materials 1. Wiley Online Library. Ch.1.

Tauc, J. 1974. Amorphous and Liquid Semiconductors. Plenum, London. 159.

Tomlin, S. G. 1968. Optical Reflection and Transmission Formulae for Thin Films. J. Phys. D: Appl. Phys. 1: 1667.

Tsuji, S., Eiji Iri and T. Hideyuki. 1992. Electronic Properties of GaAs thin film Deposited on Silica Substrate by RF Sputtering. Jpn. J. Appl. Phy. 31(3): 880-881.

Yan-Ping, YAO., LIU Chun-Ling, QAIO Zhong-Liang, LI Mei, GAO Xin and BO Bao-Xue. 2008. Structural, Optical and Electrical Properties of Hydrogen-Doped Amorphous GaAs Thin Films. Chin. Phys. Lett. 25(3): 1071.

(Received revised manuscript on 20 March, 2013) 